\title{
Microscopic reversal behavior of magnetically capped nanospheres
}

\author{
C. M. Günther, ${ }^{1,2}$ O. Hellwig, ${ }^{3}$ A. Menzel,,${ }^{1 *}$ B. Pfau, ${ }^{1,2}$ F. Radu, ${ }^{1}$ D. Makarov, ${ }^{4}$ M. Albrecht, ${ }^{4}$ A. Goncharov, ${ }^{5}$ \\ T. Schrefl,,${ }^{5,6}$ W. F. Schlotter, ${ }^{7}$ R. Rick, ${ }^{7}$ J. Lüning, ${ }^{8,9}$ and S. Eisebitt $1,2, \dagger$ \\ ${ }^{1}$ Helmholtz-Zentrum Berlin für Materialien und Energie GmbH, Hahn-Meitner-Platz 1, 14109 Berlin, Germany \\ ${ }^{2}$ Institut für Optik und Atomare Physik, Technische Universität Berlin, 10623 Berlin, Germany \\ ${ }^{3}$ Hitachi Global Storage Technologies, San Jose Research Center, San Jose, California 95135, USA \\ ${ }^{4}$ Institute of Physics, Chemnitz University of Technology, 09126 Chemnitz, Germany \\ ${ }^{5}$ Department of Engineering Materials, University of Sheffield, S1 3JD Sheffield, United Kingdom \\ ${ }^{6}$ St. Pölten University of Applied Sciences, 3100 St. Pölten, Austria \\ ${ }^{7}$ SLAC, Menlo Park, California 94025, USA \\ ${ }^{8}$ LCPMR (UMR 7614), Université Pierre et Marie Curie, 75005 Paris, France \\ ${ }^{9}$ Synchrotron SOLEIL, 91192 Gif-sur-Yvette, France
}

(Received 28 October 2009; revised manuscript received 8 January 2010; published 17 February 2010)

\begin{abstract}
The magnetic switching behavior of $\mathrm{Co} / \mathrm{Pd}$ multilayer-capped nanospheres is investigated by $\mathrm{x}$-ray spectroholography. Images of the magnetic state of individual nanocaps are recorded as a function of externally applied magnetic field and the angle under which the field is applied, pertaining to magnetic data storage applications with patterned, tilted, and perpendicular storage media. Dispersed nanospheres with different coverage in the submonolayer regime are investigated simultaneously in a multiplexed experiment. In clustered nanosphere arrangements, we find that individual switching events are influenced by dipolar magnetostatic interactions. Micromagnetic simulations of the switching behavior complement the experimental observations, corroborating the influence of thermal activation processes and magnetostatic interactions in this system. Such magnetostatic interactions could lead to undesired cross-talk between bits in ultrahigh-density magnetic recording applications.
\end{abstract}

DOI: 10.1103/PhysRevB.81.064411

PACS number(s): 75.75.Fk, 78.20.Ls, 87.59.-e, 75.78.Cd

\section{INTRODUCTION}

Arrays of magnetic nanostructures with array pitches below $100 \mathrm{~nm}$ are of large interest for magnetic data storage applications, where patterned storage media with identical repeat units promise to store one bit per individual structure, thus allowing to break the $1 \mathrm{Tbit} / \mathrm{inch}^{2}$ storage density threshold..$^{1-3}$ Many routes for reliable and cost-effective production of such storage media are currently being investigated, including top-down lithographic approaches and bottom-up self-assembly strategies. At the same time, perpendicular recording, i.e., continuous magnetic recording based on granular media with a magnetization direction perpendicular to the surface of the magnetic disk, has been implemented into customer products. ${ }^{4}$ In the development of perpendicular patterned magnetic storage media with shrinking bit sizes and array pitches from $100 \mathrm{~nm}$ toward $10 \mathrm{~nm}$, one is confronted with fundamental magnetic issues, such as the size and shape dependence of the individual magnetic properties in each prepatterned unit as well as the bit-bit interactions and the resulting magnetization reversal behavior. ${ }^{5}$ In this work, we image the switching behavior of magnetically capped nanosphere assemblies in applied magnetic fields by x-ray holography, allowing us to correlate the magnetic switching sequence with structural properties of individual nanocaps and their mutual spatial arrangement. In addition, the experimental results are compared to micromagnetic simulations taking into account magnetostatic interactions and thermal activation processes in order to understand the detailed switching behavior in external fields at finite temperatures. This information relates to the impact of nanostructure size and spatial distribution on the switching field distribution (SFD) in magnetic data storage applications and to dipolar coupling between magnetic nanostructures in general. ${ }^{6}$

\section{METHODS}

Polystyrene nanospheres of $58 \mathrm{~nm}$ average diameter and a dispersity of $8.4 \%$ are spin coated on a $\mathrm{Si}_{3} \mathrm{~N}_{4}$ window of 150 $\mathrm{nm}$ thickness and capped with a magnetic multilayer stack of $[\mathrm{Co}(3 \AA) / \operatorname{Pd}(8 \AA)]_{8}$ on a $\operatorname{Pd}(30 \AA)$ seed layer and with a $\operatorname{Pd}(12 \AA)$ antioxidation cap layer, resulting in an exchangeisolated array of magnetic caps with overall net perpendicular magnetic anisotropy. ${ }^{7-10}$ In order to follow the switching behavior by high-resolution x-ray holography, a Fourier transform holography (FTH) mask is defined on the back side of the sample using focused ion-beam patterning (FEI Strata 235) of a $1-\mu$ m-thick gold film. ${ }^{11}$ We compare the switching behavior of three sample areas, which are chosen such that they contain isolated nanospheres (region A), an approximation to a linear chain (region B), and small clusters (region $\mathrm{C}$ ) as shown in Fig. 1. As the field of view for all three sample regions is rigidly defined by the holographic mask on the back side of the membrane, we can use correlative microscopy and characterize the topography of the nanospheres and their arrangement by scanning electron microscopy (SEM, FEI Strata 235). At $10 \mathrm{kV}$ SEM voltage, the open object-defining areas in the gold film are visible from the nanosphere front side of the sample. We can thus clearly identify the microscopic field of view for the three sample areas and correlate individual nanospheres seen in SEM with 


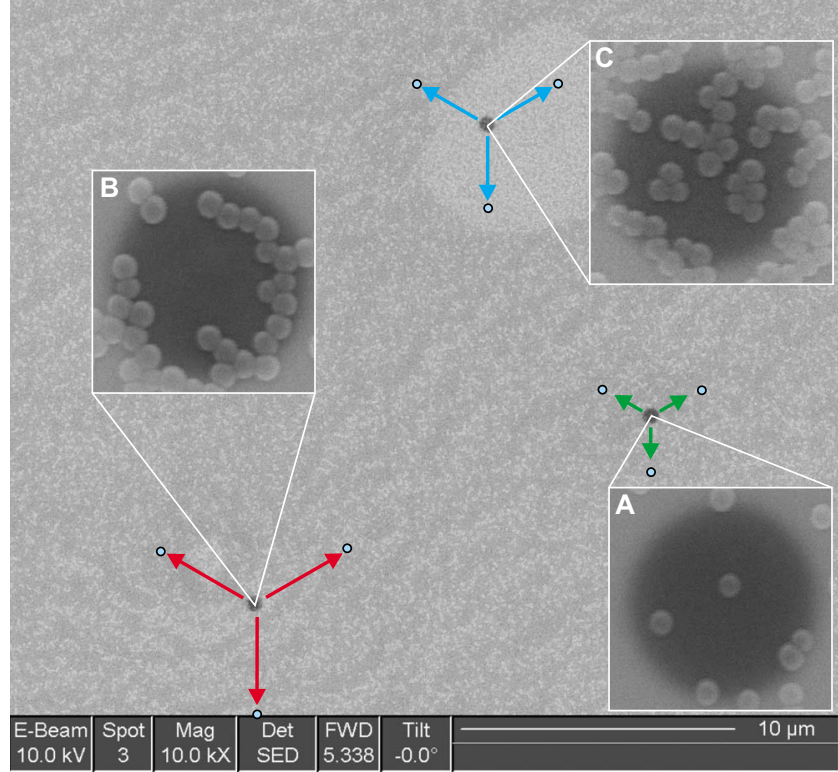

FIG. 1. (Color online) SEM image of the magnetically capped nanospheres on the holographic mask. The nanospheres are dispersed on a $\mathrm{Si}_{3} \mathrm{~N}_{4}$ membrane. The back side of the membrane is covered with a gold film in which object and reference holes have been generated at selected points. The object holes defining the three sample areas A, B, and C are visible through the membrane: insets show higher resolution SEM images of these areas, where the diameter of the circular fields of view corresponds to $\sim 590 \mathrm{~nm}$. The smaller reference holes $(\varnothing<100 \mathrm{~nm})$ are not visible in this overview image; their location has been marked by the overlaid arrows and circles. This arrangement allows for the recording of three images of each sample region simultaneously in one hologram. The multiplexed data acquisition is not only efficient, it also ensures that the magnetic field strength and direction is the same at all three sample regions.

the magnetic switching field obtained by resonant x-ray holography. In order to ensure that all three sample areas are imaged under identical conditions, x-ray holography is carried out in a multiplexed fashion, i.e., all three sample regions are contained within a $15 \mu \mathrm{m} \times 20 \mu \mathrm{m}$ region on the same FTH mask (Fig. 1) and the images of all three sample areas are encoded simultaneously in the hologram. ${ }^{12,13}$ This way, it is ensured that all three regions are exposed to the exact same magnetic field strength and field angle applied by means of an in-vacuum electromagnet.

In a spectro-holography approach, ${ }^{11,14-16}$ we use resonant scattering at the Co $L_{3}$ absorption edge with circularly polarized $\mathrm{x}$ rays in order to obtain sensitivity to the local sample magnetization via $\mathrm{x}$-ray magnetic circular dichroism (XMCD). In particular, the sample normal is parallel to the incident X-ray beam. Since the external magnetic field can be applied collinear to the incident x-ray beam or at an angle, we can study the angular dependence of the switching process, which is relevant for tilted magnetic data storage media. ${ }^{17,18}$ The magnetic state of the sample exposed to the magnetic field applied at field angles of $0^{\circ}, 30^{\circ}$, and $60^{\circ}$ with respect to the sample normal is investigated. Holograms of the nanosphere caps in the remanent state are recorded subsequently with positive and negative $\mathrm{x}$-ray helicity. Between such a pair of images, only the magnetic contrast is inverted. Difference images thus exhibit purely magnetic information, as nonmagnetic contributions, e.g., originating from thickness variations in the sample, cancel in first approximation. The holograms were recorded at room temperature in the ALICE scattering chamber $^{19}$ at the BESSY-II UE52-SGM beamline at $\lambda=1.59 \mathrm{~nm} \mathrm{X}$-ray wavelength, corresponding to the maximum XMCD contrast at the $\mathrm{Co} L_{3}$ absorption edge. The monochromator provides a longitudinal coherence in excess of $5000 \lambda$. Without further spatial filtering, transversely coherent illumination is ensured by placing the sample 300 $\mathrm{mm}$ downstream of the beamline focus, characterized by a horizontal and vertical emittance of $100 \mu \mathrm{m} \times \mathrm{mrad}$ full width half maximum each. The cumulated exposure time per hologram on the back illuminated and thinned soft x-ray charge-coupled device (CCD) camera (Princeton Instruments $2048 \times 2048$ pixels) was about 10 min per multiplexed hologram. A beamstop of approximately $200 \mu \mathrm{m}$ diameter was placed in front of the CCD in order to block residual transmitted $\mathrm{x}$ rays in the forward direction. Real-space images were obtained by a two-dimensional fast Fourier transform of the recorded holograms.

In order to get a better understanding of the reversal behavior and the underlying spin structure of the stable magnetic configurations, micromagnetic simulations at zero temperature were performed using time integration of the Landau-Lifshitz-Gilbert equation. In order to define the magnetic properties of the multilayer cap on the spherical particle, the volume of the cap was divided into a tetragonal mesh of finite elements with maximum side length of $5 \mathrm{~nm}$. As in Ref. 8, the easy axis of magnetization of each finite element is perpendicular to the nanosphere surface, leading to a tilt of the magnetic easy axis according to the curved surface of the nanosphere. Due to the surface curvature the thickness of the deposited film in radial direction becomes smaller with the inclination angle $\varphi$ from the zenith direction of the nanocap, thus influencing the magnetic properties of the nanocaps. In this case the magnitude of the magnetic anisotropy $K_{\mathrm{U}}$, saturation magnetization $M_{\mathrm{S}}$, and exchange constant $A$ of the individual elements become a function of the angle $\varphi$. While for higher angles all magnetic parameters start to decay, for $\varphi \leq 50^{\circ}$ all magnetic parameters are kept constant. ${ }^{8}$ In order to be able to investigate the magnetostatic coupling between neighboring magnetic caps, the finiteelement model for a single cap was extended to the simulation of the switching behavior of an interacting ensemble of magnetically capped nanospheres. Thermal effects in nanocaps were calculated using a nudged elastic band method (NEBM). ${ }^{20}$ These calculations were used to analyze the energy landscape for magnetic switching of the nanoscale structures by providing an optimum path between two energy states. If these two states are separated by a transition path with one or several energy minima and maxima, analysis of the path results in numerical values for the energy barriers in units of $k_{\mathrm{B}} T, T$ being the absolute temperature and $k_{\mathrm{B}}$ the Boltzmann constant. The transition frequency $f$ between two states separated by an energy barrier $E_{\mathrm{B}}$, is calculated using the Arrhenius-Néel formula. This transition frequency describes the probability of a switching event while the inverse value is a measure of the lifetime of the particular magnetization state, 

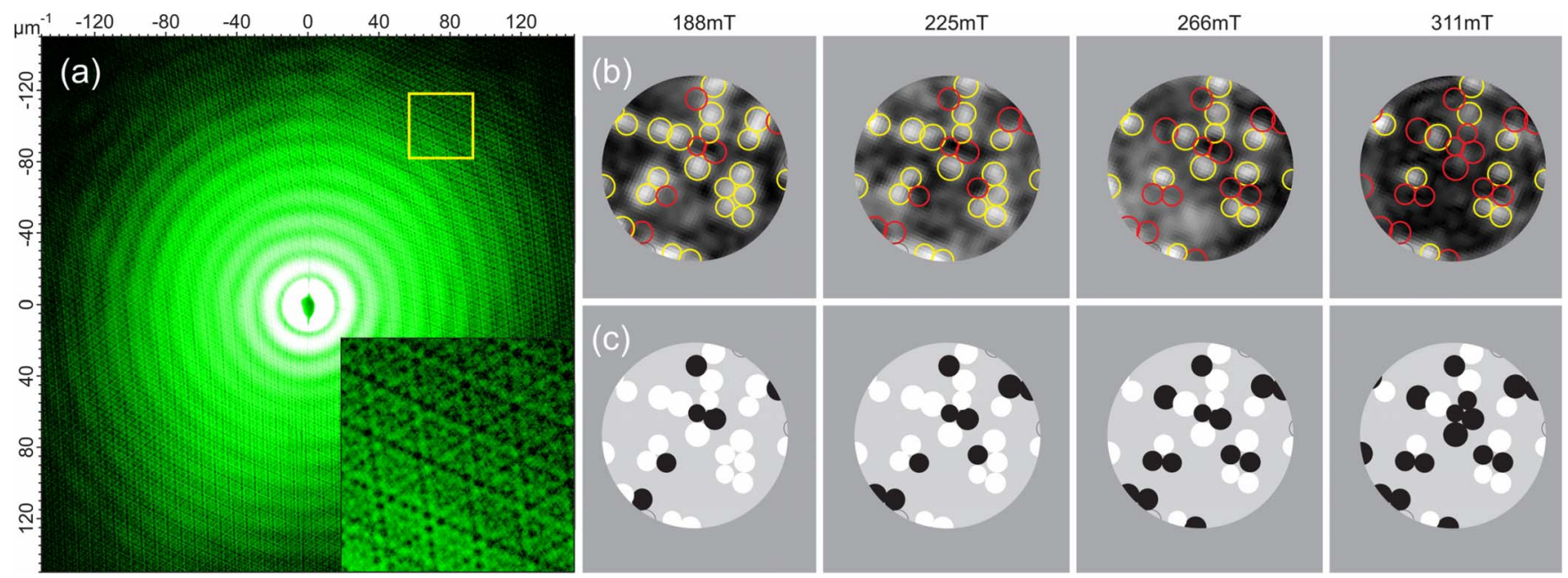

FIG. 2. (Color online) (a) Representative hologram on a logarithmic intensity scale recorded at an external magnetic field of $266 \mathrm{mT}$ with circularly polarized soft $\mathrm{x}$ rays of $1.59 \mathrm{~nm}$ wavelength. The entire hologram covers a $q$ range up to $150 \mu \mathrm{m}^{-1}$. The inset magnifies the square region around $125 \mu \mathrm{m}^{-1}$ momentum transfer, which corresponds to a real-space resolution of $50 \mathrm{~nm}$. The triangular pattern reflects the overall sample/reference geometry seen in Fig. 1 and contains the image information for all three sample regions simultaneously. (b) Reconstructed magnetization images are shown for sample region C after the application of external magnetic fields of 188, 225, 266, and $311 \mathrm{mT}$. Overlay circles are meant as a guide to the eye and were determined from SEM images shown in Fig. 1. (c) Resulting models for the magnetic state of the caps.

$$
t=\frac{1}{f_{0}} \exp \left[\frac{E_{\mathrm{B}}}{k_{\mathrm{B}} T}\right],
$$

where $f_{0}$ is the switching attempt frequency of the magnetic material. The energy barrier technique can also be used to calculate the switching field of a cap in the presence of thermal fluctuations. When an external magnetic field is applied, the energy barrier is lowered, and hence at some point the magnetization of the cap undergoes switching with assistance of thermal fluctuations.

\section{RESULTS AND DISCUSSION}

Nanospheres capped with magnetic multilayers providing perpendicular anisotropy have been shown to possess two stable states of the magnetization at remanence, namely, magnetization "up" and "down." "-10 As an example, we present a part of a switching sequence studied by spectroholography, showing one representative hologram and corresponding magnetization images as a function of the magnetic field applied normally to the sample. While each hologram [Fig. 2(a)] contains the magnetic information from all three sample regions simultaneously, the reconstructed images of the sample magnetization shown in Fig. 2(b) are for sample region $\mathrm{C}$ only. The overlay circles indicate the positions of the nanospheres as derived from the SEM images shown in Fig. 1. Note that, as hologram images show the magnetic contrast only, the caps on nanospheres are not visible if the surrounding magnetic film on the substrate has the same magnetization direction. For clarity, the magnetization state of the individual caps on the nanospheres is illustrated in a black/white model in Fig. 2(c). The magnetic switching of the caps on the nanospheres can be easily followed in the holographically generated images.

Our first observation is that, independent of the angle of the applied magnetic field, the microscopic switching behavior is highly deterministic. We erase any particular magnetic state of the array by magnetically saturating the samples, consecutively ramp to a specified opposite magnetic field and then image the resulting magnetization pattern of the array at remanence. Repeating this procedure for each of the three different angles $\left(0^{\circ}, 30^{\circ}\right.$, and $\left.60^{\circ}\right)$, we observe that among 311 individual nanocap switching events only 11 times a deviating magnetic state was observed (not shown). As polarized $\mathrm{x}$ rays are a weakly interacting magnetic probe which cannot switch the magnetization state in the system under investigation, we assume that at certain critical fields small changes in the magnetic field as well as thermal activation processes can tip the balance toward one of the two magnetically stable states of each cap, thus explaining the $4 \%$ seemingly nondeterministic events. This degree of reproducibility can be regarded as high if one considers that, unlike in storage applications where the external magnetic field is applied locally at the write-head position, we apply the external field over the entire macroscopic sample, including the regions outside the field of view. If interactions between (even just a subensemble of) nanospheres are present, local fluctuations somewhere on the sample may ultimately influence the switching sequence in the field of view. From this viewpoint, the few seemingly nondeterministic switching events may be a consequence of dipolar interactions between the bits. We will investigate this point in more detail below.

Because of the high reproducibility of the switching process, we can assign a switching field to each individual magnetically capped nanosphere by inspecting holographic images of the magnetization at nine consecutive points of a remanent hysteresis loop, where each magnetic field value is approached from saturation. The particle-resolved magnetic switching fields are presented in Fig. 3 by a color coded 


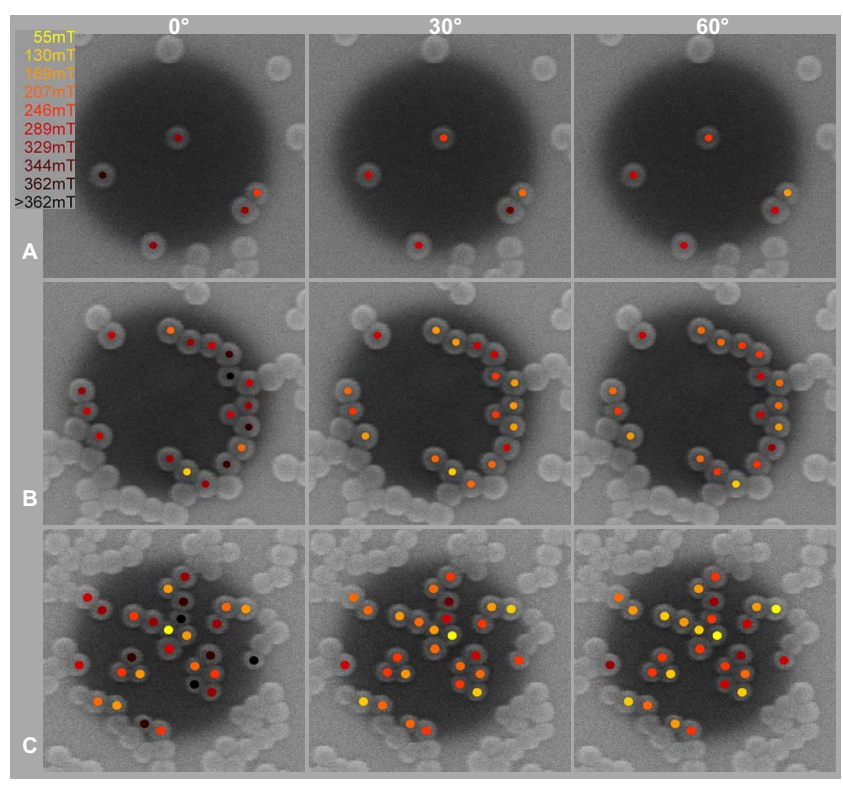

FIG. 3. (Color online) Correlative images of the capped nanosphere topography imaged by SEM and the switching field values for individual caps determined by spectro-holography (color dot overlay). The different nanosphere arrangements of sample regions $\mathrm{A}, \mathrm{B}$, and $\mathrm{C}$ are presented in the rows. The columns correspond to the microscopic switching fields measured for three included angles between the sample normal and the direction of the externally applied magnetic field at $0^{\circ}, 30^{\circ}$, and $60^{\circ}$.

overlay of the SEM images. The switching information is compiled from stacks of multiplexed holographic images at nine magnetic fields for each of the three geometries.

The average switching behavior in a macroscopic ensemble of magnetic caps has been reported earlier as a function of nanosphere size and the angle between the external field and the sample normal. ${ }^{8}$ Deviating from StonerWohlfarth model predictions ${ }^{21,22}$ no subsequent increase in the switching field was found for angles larger than $45^{\circ}$. Instead, for 50-nm-diameter nanospheres, the switching field decreased monotonically up to an angle of $60^{\circ}$, where a plateau extending up to $80^{\circ}$ was reached. Micromagnetic simulations explained the deviation from a Stoner-Wohlfahrt reversal mechanism as being due to the angular distribution of the easy magnetic axis within each cap on a nanosphere. ${ }^{8}$

Combining magnetic information from spectroholography with topography information obtained by SEM, Fig. 3 summarizes the switching fields for three directions, in which the magnetic field is applied. When the field is applied perpendicular to the sample, differences in the switching fields of up to $310 \mathrm{mT}$ between different magnetically capped nanospheres are observed, as several magnetic caps on nanospheres turn out to be very hard to switch. An angle between the sample normal and the field direction clearly reduces the observed differences in the switching field. Remanent magnetization curves, measured exclusively on the magnetic caps on the nanospheres, are presented in Fig. 4. These curves were derived from the magnetization images, taking into account the projected surface area of each nanosphere along the macroscopic sample normal. The magnetic field for which $50 \%$ of magnetic caps of the complete en-

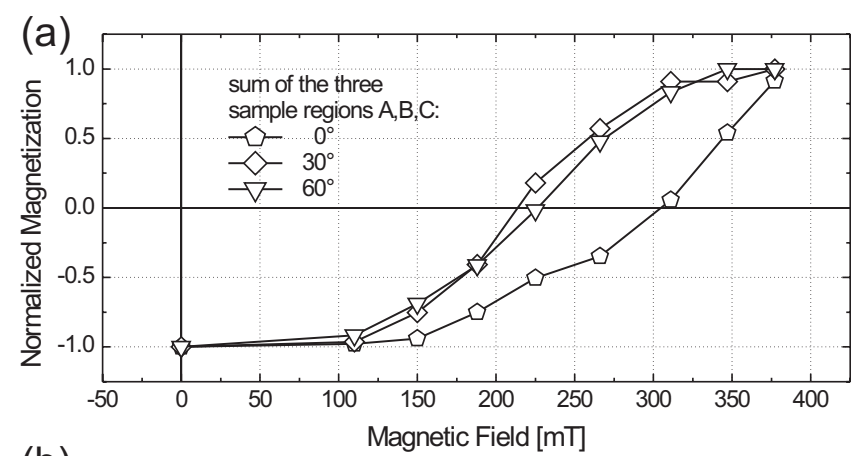

(b)

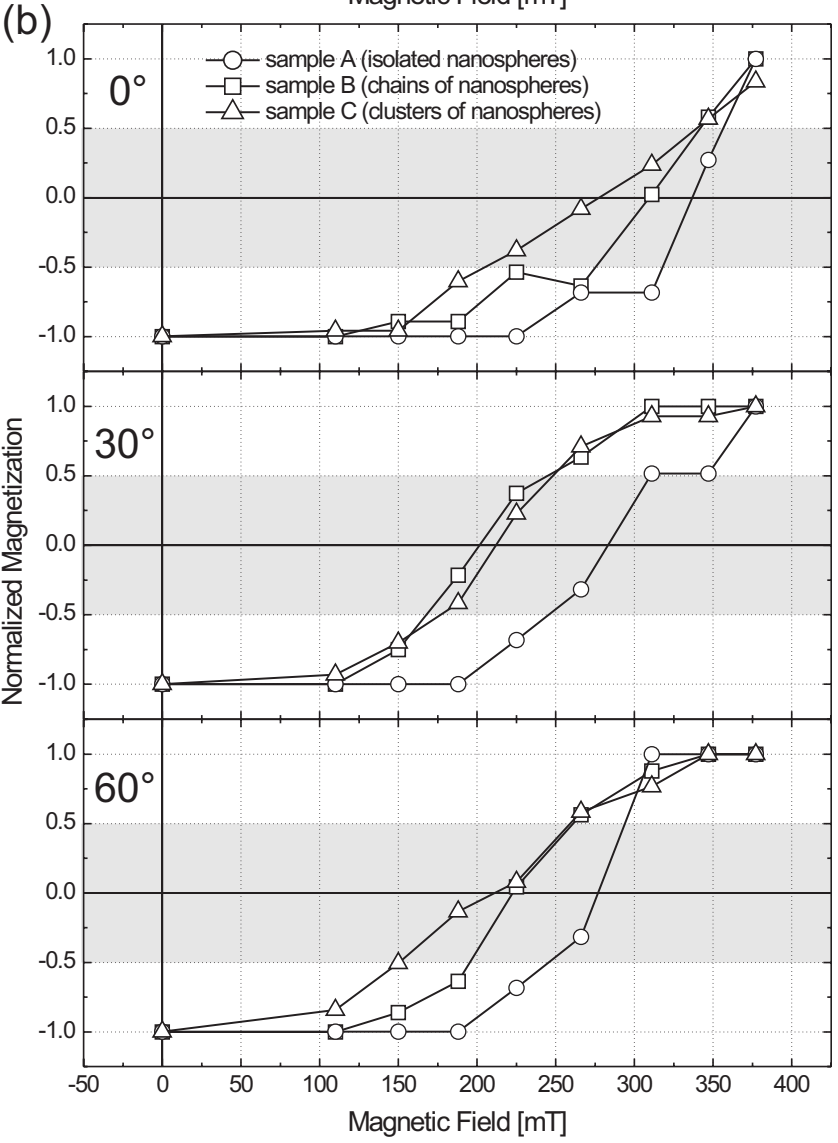

FIG. 4. Remanent magnetization curves for the nanocaps obtained from images recorded at remanence after applying a reverse magnetic field to the initially saturated sample. For each magnetic field value the sizes of the nanocaps are summed up with a sign corresponding to their magnetic state. The curves correspond to one branch of a complete hysteresis loop. In (a) we show the hysteresis branches of all three sample regions, A, B, and C, together for the angles of $0^{\circ}, 30^{\circ}$, and $60^{\circ}$. In (b) the contribution of each sample region is plotted separately to investigate magnetostatic effects. The gray area marks the magnetization interval from which the SFD width is determined.

semble reversed their magnetization is reduced from $310 \pm 20 \mathrm{mT}$ at $0^{\circ}$ to $220 \pm 20 \mathrm{mT}$ and $230 \pm 20 \mathrm{mT}$ at $30^{\circ}$ and $60^{\circ}$, respectively [Fig. 4(a)]. The errors in the magnetic field are dominated by the magnetic field intervals at which images were recorded. For lithographically fabricated, monodisperse arrays of nanopillars covered with $\mathrm{Co} / \mathrm{Pd}$ multilayers similar to the ones used here (but without the anisotropy gradient introduced by the spherical surface of the 
nanospheres), the width of the switching field distribution was found to be dominated by intrinsic variations in the local magnetic anisotropy. For islands with 50 and $100 \mathrm{~nm}$ diameter, the width of the SFD was found to be approximately 150 and $130 \mathrm{mT}$, with maxima at 630 and $550 \mathrm{mT}$, respectively. ${ }^{5}$ A mix of both sizes would result in a SFD with a width of approximately $200 \mathrm{mT}$, which compares reasonably with our values on a small ensemble with considerable size variation and disorder. The reduction in the switching field at $30^{\circ}$ and $60^{\circ}$ compared to $0^{\circ}$ observed in the present study is in good agreement with previously reported data on close-packed multilayer covered nanosphere arrays. ${ }^{8}$

A first indication for magnetostatic coupling between different nanocaps stems from the comparison of the magnetization curves in Fig. 4(b) as a function of the cap geometries. For the nanospheres which are part of clusters (sample region $\mathrm{C}$ ) or linear chains (sample region $\mathrm{B}$ ) we observe a broadened switching interval as compared to isolated nanospheres (sample region A). Note, that such an increase in the width of the switching field interval is observed in the experiments at $0^{\circ}$ and $60^{\circ}$ but not at $30^{\circ}$ probably as a result of the statistical error due to the limited number of data points. While results on dipolar interactions between nanostructures depend greatly on the individual structures and their arrangement, a broadened SFD is generally expected for the prototypical situation of two magnetic moments with their easy axis perpendicular to the separation vector connecting the nanostructures on which the moments reside. ${ }^{6}$ As experimental findings on the influence of magnetostatic coupling in nanostructure arrays reported in the literature depend crucially on the type and arrangement of the nanostructures, ${ }^{6}$ we focus on a deeper understanding of the behavior observed for our particular arrangement of magnetic nanocaps.

Inspection of the coercivity field of individual magnetic nanocaps, which are part of a nanosphere ensemble in the sample areas A (isolated), B (chain), and C (cluster), shows deviations from the size dependence of the reversal fields expected for isolated caps. In particular, we find caps with approximately similar diameter that switch at significantly different fields and smaller-diameter caps which switch at lower fields than larger-diameter caps (Fig. 3). These findings differ from experimental results reported for dense monodisperse nanocap arrays with different particle sizes. ${ }^{7}$

The latter is an indication that thermal activation processes as well as magnetostatic interactions have to be taken into account. In order to better understand the behavior in our particular ensembles of nanocaps, micromagnetic simulations were carried out for the clustered nanospheres in sample region $\mathrm{C}$. Diameters and relative arrangement of the nanospheres were obtained from SEM images and transferred into the model. Note that the model represents an idealized situation as several capped nanospheres appear flattened at the contact line to neighboring spheres in the SEM image. In such situations, the center position of the sphere has been retained for the model and the diameters have been adjusted in steps of $5 \mathrm{~nm}$ so that spheres do not intersect. The magnetic parameters used for the simulations were $M_{\mathrm{S}}$ $=0.5 \mathrm{~T}, A=1 \times 10^{-11} \mathrm{~J} / \mathrm{m}$, and $K_{\mathrm{U}}=0.125 \mathrm{MJ} / \mathrm{m}^{3}$ with the angular dependence described in the methods section. A series of snapshots of the magnetization distribution of the re-
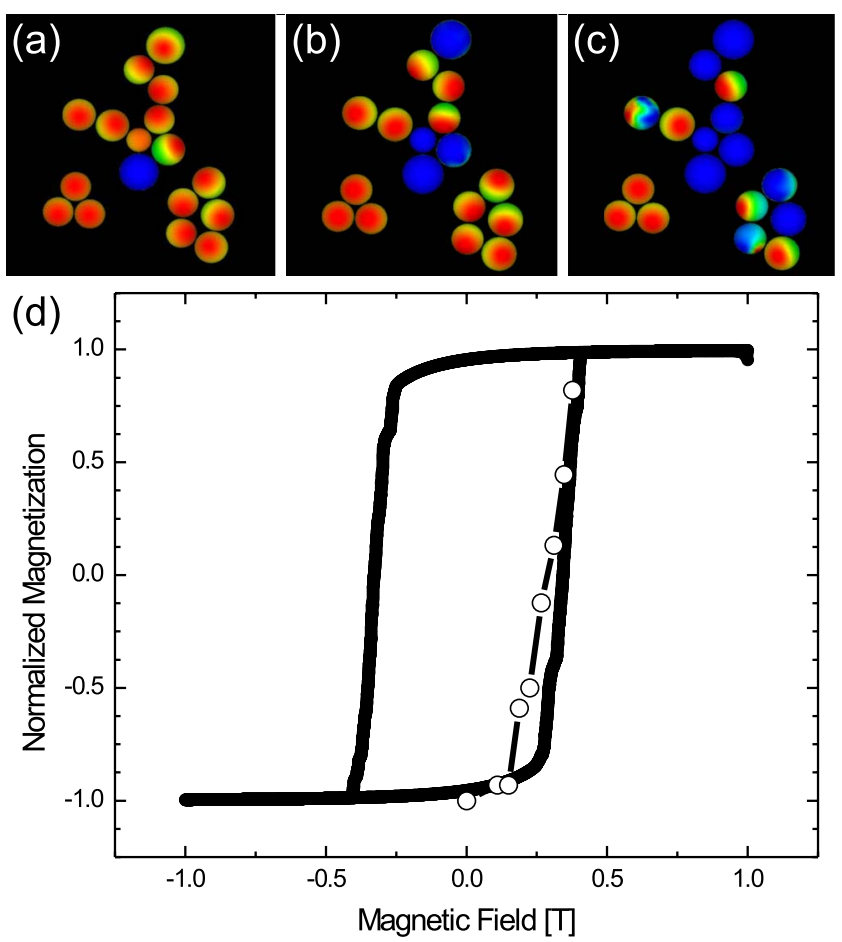

FIG. 5. (Color online) Snapshots of the magnetization state of a cap array within sample region $\mathrm{C}$. Results from the micromagnetic simulations are shown for an applied magnetic field of (a) $270 \mathrm{mT}$, (b) $320 \mathrm{mT}$, and (c) $340 \mathrm{mT}$ at zero temperature. The magnetic field was applied normal to the sample. Red and blue indicate maxima $( \pm 1)$ of the magnetization component normal to the sample and green indicates that this component is zero. (d) The calculated hysteresis loop (black line) is compared to the hysteresis branch (white circles) derived from holographic imaging, taking the exact same nanocap subset within sample area $\mathrm{C}$ into account.

gion $\mathrm{C}$ array in the applied reversed magnetic fields is shown in Figs. 5(a)-5(c). The switching of nanocaps of the same size at different fields is visible, as well as the existence of small particle caps that switch before larger ones reverse their magnetization [see Fig. 5(b)]. Thus, effects of the magnetostatic coupling on the reversal behavior are apparent, in agreement with the experimental results summarized in Fig. 3. The $M(H)$-hysteresis loop for the nanocap array in Figs. 5(a)-5(c), as obtained from the micromagnetic simulations, is presented in Fig. 5(d). We find in the simulations that the broadening of the SFD of approximately $160 \mathrm{mT}$ is mainly caused by the particles' size distribution and magnetostatic coupling between the caps. The presence of defects, pinning centers, and thermal activation processes, excluded from the present micromagnetic simulations, would lead to further broadening in SFD. This is in line with the experimentally observed SFD from the same nanocap subset within area C, which is approximately $200 \mathrm{mT}$ at room temperature.

Micromagnetic simulations at zero temperature explain the switching field distribution caused by the size distribution and magnetostatic coupling. In order to include thermal activation processes, which were neglected so far, energy barriers at room temperature for caps were analyzed using NEBM simulations. This method proved to be very successful to select the minimum energy path, also in situations 


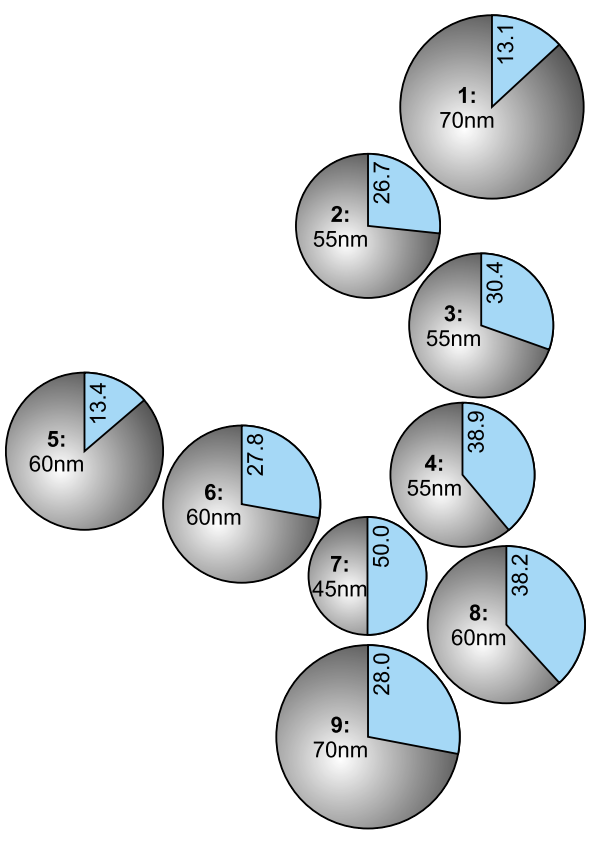

FIG. 6. (Color online) Results of the energy barrier simulations have been overlaid on the individual caps for which the result was obtained. The difference in the energy barrier for each cap is calculated for two cases: all neighbors magnetized in up direction and all neighbors magnetized in down direction. These two configurations provide most destabilizing and stabilizing dipolar fields for the target cap. The resulting energy barrier difference is normalized to the energy barrier of the corresponding cap in zero field. The size of the pie indicates the result of this calculation for the respective cap, indicating the influence of magnetostatic interactions on the switching field at finite temperature. For clarity, the cap number, the diameter used in the simulations and $d E / E$ in percent is also denoted at each cap.

where more than one path can connect different minima. An example is the correct selection of one of three different paths for reversal in magnetic random memory cells depending on their size..$^{23}$ For the relaxation method toward the minimum energy path we use the implementation described in Ref. 24. Ambiguities are avoided as the path is always rotated into a plane spanned by the initial magnetization and any external field acting on the initial state. Dipolar interactions between caps were included in simulations using a boundary element method. To study the effect of coupling on the SFD only 9 caps were used, shown in Fig. 6. Two other small groups of caps within region $\mathrm{C}$ were neglected because their magnetostatic field does to good approximation not influence the region of interest due to the large separation distance. In Fig. 6 caps are numbered from 1 to 9, with diameters distributed as follows: $70 \mathrm{~nm}$-caps with numbers 1 and 9, $55 \mathrm{~nm}$ - caps with numbers 2,3 , and 4, $60 \mathrm{~nm}$ - caps with numbers 5,6 , and $8,45 \mathrm{~nm}$ - the cap with number 7 . For each cap the energy barrier for the transition from an up to down magnetized state was calculated for two extreme scenarios: first all other neighbors were considered to be magnetized up and in the second scenario they were magnetized in the down direction. From the results of these simulations we obtain the maximum possible spread in the energy barrier caused by dipolar fields. The difference in the energy barrier between the two scenarios has been included in Fig. 6 in the form of a pie chart superimposed on the position of the respective cap. The values are normalized to the respective energy barriers at zero field. Thus the results reflect the change in the energy barrier and consequently are a measure of the nonzero temperature switching field owing to the magnetostatic interactions only. Size effects are excluded by normalizing with the energy barrier at zero interaction field of the cap under consideration. It is obvious from this figure that the size of this magnetostatic contribution to the switching correlates with the number of close neighbor caps. We conclude that despite of the complex situation with a curved surface and an azimuthally dependent anisotropy on each nanocap, the overall behavior with respect to dipolar interaction is quite well approximated by a simple picture of one effective magnetic moment per cap with an easy axis perpendicular to the intercap separation vectors. Nevertheless, details of the switching will depend on the switching dynamics. We find in the simulations that the computed minimum energy path is a nonuniform rotation of the magnetization.

Note that the normalized magnetostatic contribution does not relate directly to the switching sequence of the nanocaps due to the size and zero-field normalization and due to the specific magnetostatic situation encountered at each step of a particular switching sequence. Apart from providing general insight into the magnetostatic situation for a particular cap configuration, we note that the calculations help to rationalize, e.g., the unusual behavior seen in the first switching step of array $\mathrm{C}$ under $0^{\circ}$ shown in Fig. 3: apparently the smallest nanosphere cap no. 7 switches first in the external field due to the large magnetostatic influence as indicated in Fig. 6. Clearly, the relative importance of the magnetostatic interactions is reduced as soon as a tilted-media configuration with an included angle between the external field and the sample normal is realized.

\section{SUMMARY}

We have used x-ray spectro-holography to image the magnetization state of magnetic nanocap arrays. The obtained data on the magnetization state is combined with highresolution scanning electron microscopy images of the sample topography in a correlative microscopy approach. The microscopic switching behavior after the application of external magnetic fields was followed as a function of field strength, field angle, and the sample topography (isolated, chain, and clustered cap assemblies). The magnetic switching behavior of the nanocaps is highly deterministic, allowing us to assign angle-dependent switching fields to individual nanocaps. We observe lower switching fields and a narrowing of the switching field distribution with an included angle between the macroscopic sample normal and the externally applied magnetic field, in agreement with earlier measurements on macroscopic ensembles of nanospheres. For magnetic cap structures which have direct neighbors as part of a chain or cluster, we find evidence for a magnetostatic influence of the surrounding nanocaps on the switching behavior, most apparent for caps of the same size in different neighbor geometries. In particular, we observe 
smaller-diameter nanocaps which switch at lower magnetic fields than larger nanocaps. We have carried out micromagnetic simulations of the energy landscape and switching behavior for a particular nanocap array including thermal effects. The simulations qualitatively reproduce our experimental results and show that magnetostatic interactions in the array and thermal activation have to be taken into account in addition to the properties of the individual nanocaps in order to understand the detailed switching behavior in external fields at finite temperatures. In a magnetic data storage context, these magnetostatic interactions would be experienced as cross-talk between individual bits. With further shrinking bit sizes and bit pitches for future storage densities in the terabit per square inch regime, the influence of magnetostatic bit-bit interactions will thus become increasingly relevant for the bit switching behavior.

\section{ACKNOWLEDGMENTS}

We thank H. Zabel for providing the ALICE scattering chamber. In addition to the general support from the BESSY-II staff, we benefited in particular from the beam block adjustment designed by T. Noll. A.M. acknowledges EU support under Contract No. MTKD-CT-2004-003178.
*Present address: Paul Scherrer Institut, CH-5232 Villigen, Switzerland.

${ }^{\dagger}$ Corresponding author; eisebitt@ physik.tu-berlin.de

${ }^{1}$ M. L. Plummer, J. van Ek, and D. Weller, The Physics of UltraHigh-Density Magnetic Recording (Springer, Berlin, 2001).

${ }^{2}$ A. Moser, K. Takano, D. T. Margulies, M. Albrecht, Y. Sonobe, Y. Ikeda, S. H. Sun, and E. E. Fullerton, J. Phys. D 35, R157 (2002).

${ }^{3}$ B. D. Terris and T. Thomson, J. Phys. D 38, R199 (2005).

${ }^{4}$ S. N. Piramanayagam, J. Appl. Phys. 102, 011301 (2007).

${ }^{5}$ T. Thomson, G. Hu, and B. D. Terris, Phys. Rev. Lett. 96, 257204 (2006).

${ }^{6}$ J. I. Martín, J. Nogués, K. Liu, J. L. Vicent, and I. K. Schuller, J. Magn. Magn. Mater. 256, 449 (2003), and references therein, esp. in Sec. 3.2.

${ }^{7}$ M. Albrecht, G. H. Hu, I. L. Guhr, T. C. Ulbrich, J. Boneberg, P. Leiderer, and G. Schatz, Nature Mater. 4, 203 (2005).

${ }^{8}$ T. C. Ulbrich, D. Makarov, G. Hu, I. L. Guhr, D. Suess, T. Schrefl, and M. Albrecht, Phys. Rev. Lett. 96, 077202 (2006).

${ }^{9}$ T. C. Ulbrich, D. Assmann, and M. Albrecht, J. Appl. Phys. 104, 084311 (2008).

${ }^{10}$ P. Kappenberger, F. Luo, L. J. Heyderman, H. H. Solak, C. Padeste, C. Brombacher, D. Makarov, T. Ashworth, L. Philippe, H. J. Hug, and M. Albrecht, Appl. Phys. Lett. 95, 023116 (2009).

${ }^{11}$ S. Eisebitt, J. Lüning, W. F. Schlotter, M. Lörgen, O. Hellwig, W. Eberhardt, and J. Stöhr, Nature (London) 432, 885 (2004).

${ }^{12}$ W. F. Schlotter, R. Rick, K. Chen, A. Scherz, J. Stöhr, J. Lüning,
S. Eisebitt, Ch. Günther, W. Eberhardt, O. Hellwig, and I. McNulty, Appl. Phys. Lett. 89, 163112 (2006).

${ }^{13}$ W. F. Schlotter, J. Lüning, R. Rick, K. Chen, A. Scherz, S. Eisebitt, C. M. Günther, W. Eberhardt, O. Hellwig, and J. Stöhr, Opt. Lett. 32, 3110 (2007).

${ }^{14}$ O. Hellwig, S. Eisebitt, W. Eberhardt, W. F. Schlotter, J. Lüning, and J. Stöhr, J. Appl. Phys. 99, 08H307 (2006).

${ }^{15}$ S. Eisebitt, M. Lörgen, W. Eberhardt, J. Lüning, and J. Stöhr, Appl. Phys. A: Mater. Sci. Process. 80, 921 (2005).

${ }^{16}$ S. Eisebitt, M. Lörgen, W. Eberhardt, J. Lüning, J. Stöhr, C. T. Rettner, O. Hellwig, E. E. Fullerton, and G. Denbeaux, Phys. Rev. B 68, 104419 (2003).

${ }^{17}$ J. P. Wang, Y. Y. Zou, C. H. Hee, T. C. Chong, and Y. F. Zheng, IEEE Trans. Magn. 39, 1930 (2003).

${ }^{18}$ J.-P. Wang, Nature Mater. 4, 191 (2005).

${ }^{19}$ J. Grabis, A. Nefedov, and H. Zabel, Rev. Sci. Instrum. 74, 4048 (2003).

${ }^{20}$ R. Dittrich, T. Schrefl, D. Suess, W. Scholz, H. Forster, and J. Fidler, J. Magn. Magn. Mater. 250, L12 (2002).

${ }^{21}$ E. C. Stoner and E. P. Wohlfarth, Philos. Trans. R. Soc. London, Ser. A 240, 599 (1948).

${ }^{22}$ R. Dittrich, G. H. Hu, T. Schrefl, T. Thomson, D. Suess, B. D. Terris, and J. Fidler, J. Appl. Phys. 97, $10 J 705$ (2005).

${ }^{23}$ R. Dittrich, T. Schrefl, H. Forster, D. Suess, W. Scholz, and J. Fidler, IEEE Trans. Magn. 39, 2839 (2003).

${ }^{24}$ D. Suess, S. Eder, J. Lee, R. Dittrich, J. Fidler, J. W. Harrell, T. Schrefl, G. Hrkac, M. Schabes, N. Supper, and A. Berger, Phys. Rev. B 75, 174430 (2007). 